\title{
Pre-filled Oral Syringe
}

National Cancer Institute

\section{Source}

National Cancer Institute. Pre-filled Oral Syringe. NCI Thesaurus. Code C149862.

Filled container for administering a liquid or semi-solid pharmaceutical form to the oral cavity, containing the final preparation in one compartment, or the components necessary for its preparation in different compartments. 